\title{
Security Analysis of Regional Integrated Energy System Integrating Energy Storage
}

\author{
Kaicheng Liu ${ }^{1}$, Ying $\mathrm{Guo}^{2}$, Dan Wang ${ }^{2, *}$, Dezhi $\mathrm{Li}^{1}$, Guixiong $\mathrm{He}^{1}$ \\ ${ }^{1}$ State Key Laboratory of Power Grid Safety and Energy Conservation Editorial Department, China Electric Power Research Institute, \\ Beijing, China \\ ${ }^{2}$ Key Laboratory of Smart Grid of Ministry of Education, Tianjin University, Tianjin, China
}

\begin{abstract}
Regional integrated energy system (RIES) can realize multi-energy conversion and complementation so as to improve energy efficiency, which also brings more security risks. The regional integrated energy system security region (RIESSR) is a security analysis method to describe the safe area for the operating points of RIES based on the N-1 guideline. As a controllable device, energy storage (ES) which is installed in the energy hub (EH) plays an important role in improving system security. Therefore, this paper establishes the model of practical security region of RIES integrating energy storage, and studies the impact of ES on total supply capability (TSC) and practical security boundary. Finally, a specific case is set to simulate and verify the model. By comparing the scenario with ES and the scenario without ES, it can be seen that the solution result of TSC increases and the security region extends across quadrant when the RIES is integrated with ES system. The capacity and location of the ES also impact on TSC and RIESSR.
\end{abstract}

\section{Introduction}

Efficient and low-carbon energy consumption is the pursuit of the world, and regional integrated energy systems is an effective way to achieve the above goal. However, multi-energy coupling makes system security face more new challenges [1].

At present, the safety analysis of RIES is mostly based on the traditional and time-consuming 'point-wise' simulation method. Security region method is developed on the basis of the 'point-wise' simulation method, which describes the area where the whole system can operate reliably and safely [2]. Reference [3-4] proposed a supply capability model for distribution systems based on $\mathrm{N}-1$ guideline and a kind of full-dimensional direct observation method of distribution system security region. Reference [5] proposed the supply capability model and security region observation method for integrated energy system.

However, existing research does not analyse the impact of ES on security region of RIES in depth. ES device is charged during the low load period and discharged during the peak load period, which will play a positive role on RIESSR. ES can be divided into centralized and distributed, among which centralized energy storage is more controllable and widely used in RIES [6]. Reference [5] predicted the expansion of the security region when the RIES is integrated with energy storage system, but did not analyse it in depth or verify it with a case.In order to solve the above research gap, this paper establishes the model of RIESSR integrated with ES, and analyses the impact of ES on RIESSR.
The rest of this paper is organized as follows. Section 2 builds the model of RIESSR integrated with ES and proposes the security constraints of key equipment and pipelines. Section 3 proposes the process of solution the practical security boundary. Section 4 simulates the RIESSR with a case. Section 5 presents the conclusion.

\section{Model of RIESSR integrated with energy storage}

RIESSR is a collection of all operating points that can pass the N-1 verification. The model of RIESSR is referred from reference [5], which can be expressed as:

$$
\Omega_{\text {RIESSR }}=\{\boldsymbol{L} \mid \boldsymbol{E}(\boldsymbol{L})=\mathbf{0}, \boldsymbol{I N}(\boldsymbol{L}) \leq \mathbf{0}\}, \quad \boldsymbol{L}=\left\{L_{1}, \cdots, L_{M}\right\}
$$

where $\left\{L_{1}, \cdots, L_{M}\right\}$ represents loads of pipelines; $\boldsymbol{E}(\boldsymbol{L})=\mathbf{0}$ is the equality constraints which represents energy flow balance; $\boldsymbol{I N}(\boldsymbol{L}) \leq \mathbf{0}$ is the inequality constraints which includes $N-1$ guideline of key equipment and key pipelines in $\mathrm{EH}$.

Operation constraints of energy storage system have been studied [7] and will not be described in detail here.

$$
C_{E S}=P_{c} \cdot \eta_{c}=P_{d} \cdot \eta_{d}
$$

where $C_{E S}$ represents the capacity of ES; $P_{c} 、 P_{d}$ and $\eta_{c} 、 \eta_{d}$ represent the charge and discharge power and efficiency coefficients.

In this paper, key equipment in $\mathrm{EH}$ is consist of $\mathrm{ES}$, transformer, CHP, gas boiler, circulation pump and compressor. When the key equipment in $\mathrm{EH}$ is out of

* Dan Wang: wangdantjuee@tju.edu.cn 
operation due to a failure, the load in the failure area will be transferred to the interconnected key equipment. And the N-1 security constraint is caused by the process of load transfer, which can be expressed as:

$$
\begin{aligned}
& L_{i j}^{\text {transfer }}+L_{j}^{\text {original }} \leq k C_{j}+C_{E S . j}+C_{E S . i} \\
& L_{j}^{\text {original }}=\sum_{x \in \Phi_{j}} L_{x}
\end{aligned}
$$

where $L_{i j}^{\text {transfer }}$ represents the load transferred from the key equipment $i$ to equipment $j$ due to the failure of $i$ or the pipelines connected to it; $L_{j}^{\text {original }}$ represents the original load supplied by equipment $j ; C_{j}$ represents the capacity of equipment $j ; k$ is the coefficient of overload and the value is 1 in this paper; $C_{E S . j}, C_{E S . j}$ represent the discharge power of ES connected to equipment $i$ and $j$, and it takes a negative value when charging; $\Phi_{j}$ represents the set of key pipelines connected to $j ; L_{x}$ represents the load supplied by pipeline $x$.

Key pipelines in EH are located at the energy supply equipment outlet. When a key pipeline is out of operation due to N-1 contingency, the load supplied by it is transferred to other interconnected key pipelines. Therefore, the constraint can be expressed as:

$$
L_{m n}^{\text {transfer }}+L_{n}^{\text {original }} \leq C_{n}+C_{E S . m}
$$

where $L_{m n}^{\text {transfer }}$ represents the load transferred from the key pipeline $m$ to the key pipeline $n$ due to the failure of $m$ or the equipment connected to it; $L_{n}^{\text {original }}$ represents the original load supplied by pipeline $n ; C_{n}$ represents the rated capacity of pipeline $n ; C_{E S . m}$ represents the discharge power of ES connected to $m$.

\section{Solution of practical security boundary integrated with energy storage}

In order to simplify the model, the non-linear operation constraints are not taken into consideration because the value of voltage, pressure drop are little because the pipelines are usually not long. And equipment such as static var compensators, compressors and circulating pumps can adjust the parameters above [8].

\subsection{Model of practical security boundary integrated with energy storage}

After simplification, the model of practical security boundary considering ES can be expressed as:

$$
\begin{aligned}
B_{m}^{\mathrm{U}}= & \min _{m \in \Phi_{i}, n \in \Phi_{j}}\left(k C_{j}+C_{E S . j}+C_{E S . i}-L_{i j}^{\text {transfer }}-L_{j}^{\text {original }},\right. \\
& \left.C_{n}+C_{E S . m}-L_{m n}^{\text {transfer }}-L_{n}^{\text {original }}, X_{n}\left(L_{m n}^{\text {transfer }}, L_{n}^{\text {original }}\right)\right)
\end{aligned}
$$

where $B_{m}^{\mathrm{U}}$ represents the upper boundary of pipeline $m$; $X_{n}\left(L_{m n}^{\text {transfer }}, L_{n}^{\text {original }}\right)$ represents additional constraint when $n$ is multi-energy coupling equipment.

$$
B_{m}^{\mathrm{L}}=\max \left(L_{m n}^{\text {transfer }}+L_{n}^{\text {original }}-P_{E . m}, L_{m n}^{\text {transfer }}+L_{n}^{\text {original }}-0\right)
$$

where $B_{m}^{\mathrm{L}}$ represents the lower boundary of pipeline $m$; $P_{E . m}$ represents power supplied by pipeline $m$ and consumed by compressors or circulation pumps, which is additional constraint when $m$ supplies energy to above equipment. It is worth noting that the new state $-C_{E S . m} \leq L_{m} \leq 0$ represents a special operating condition in which the ES is charged by the other energy hub, and the operating point is safe considering the controllability of ES charge and discharge state.

\subsection{Solution process of practical security boundary integrated with energy storage}

There are three steps in the solution process of practical security boundary integrated with ES. The first step is to solve TSC and record the operating point at TSC which describes the load of each key pipelines. The second step is to choose free variables and solve critical operating points. The third step is to fit the critical operating points and get the security boundary. The solution process of practical security boundary integrated with ES is shown in Fig. 1 . 


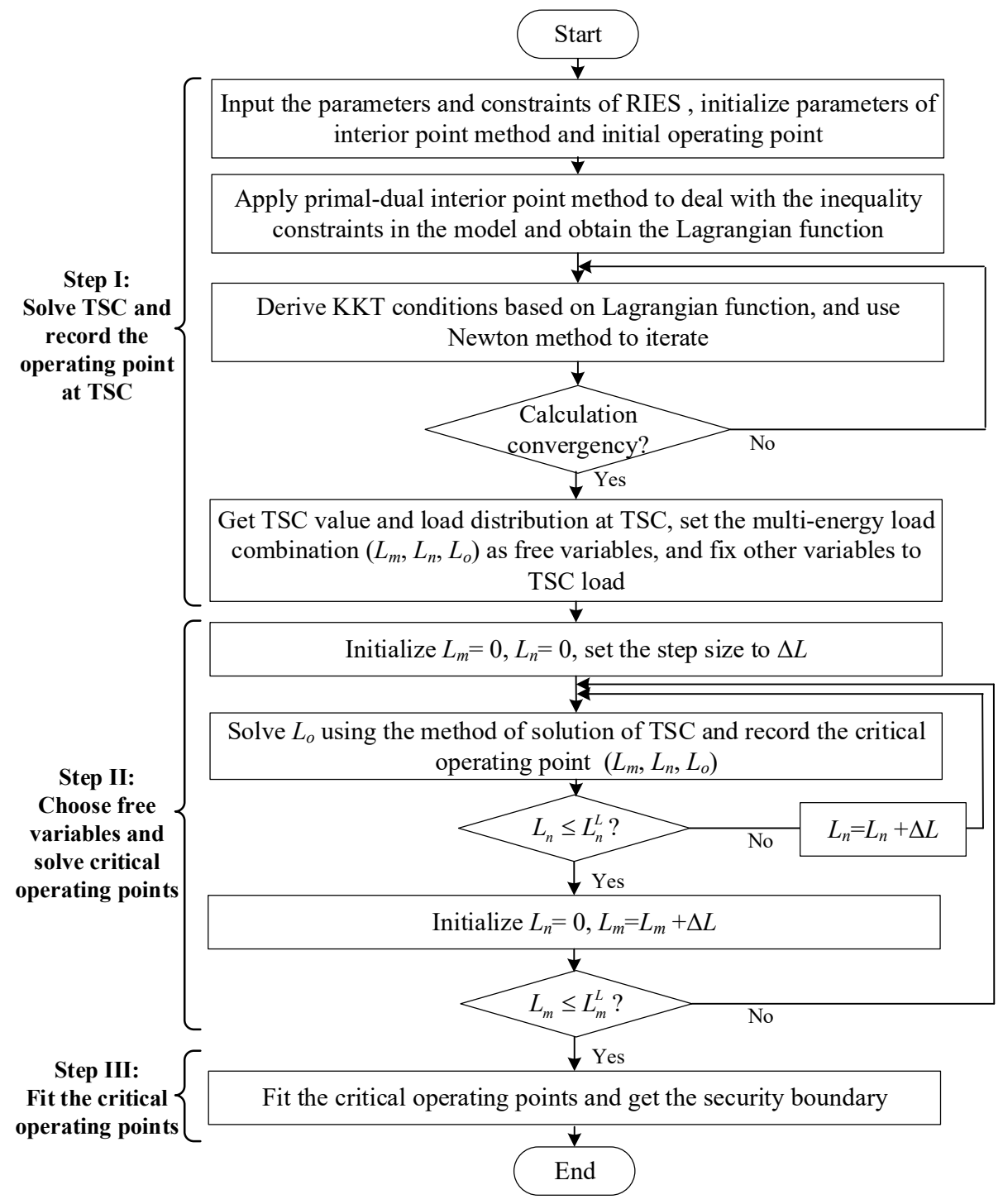

Figure 1. The solution process of practical security boundary

\section{Case study}

\subsection{Overview of base case}

The base case is a typical RIES integrated with ES. And the multi-energy coupling equipment include CHP, gas

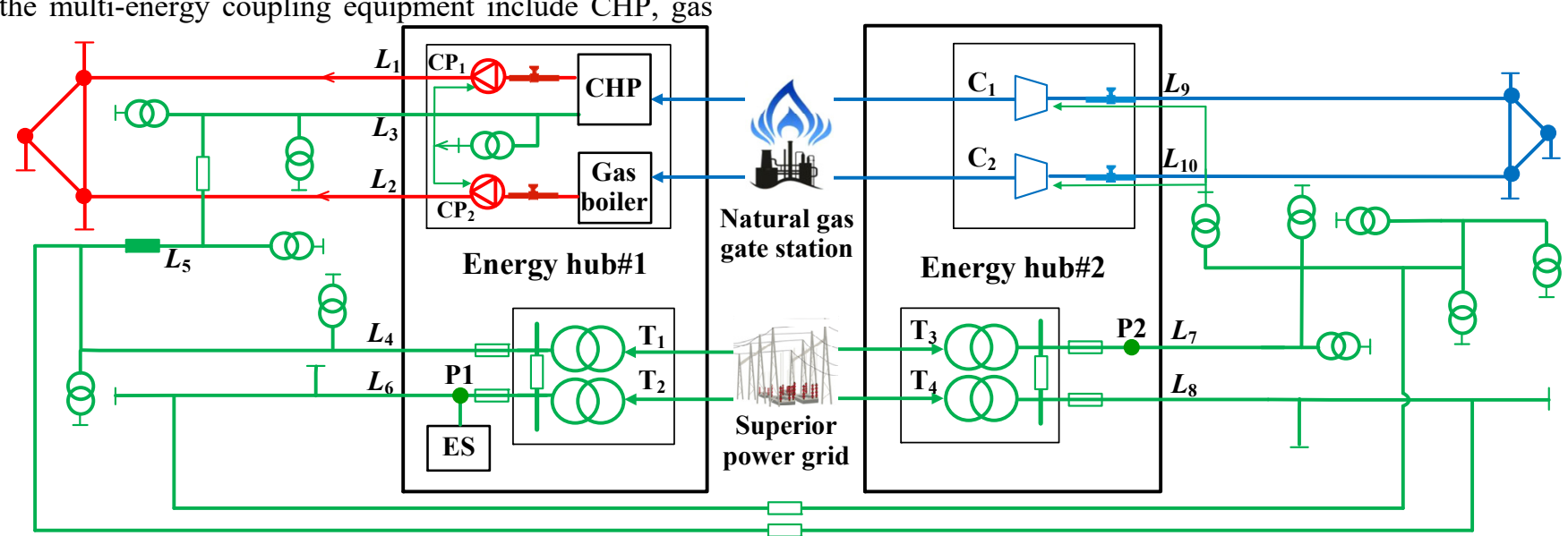

$\sqcup$ Feeder switch $\square$ Sectionalizing switch $\square$ Tie switch $\simeq$ Valve $Q$ Circulation pump $\longrightarrow$ Natural gas $\longrightarrow$ Heat $\longrightarrow$ Electricity $Q$ Transformer $\square$ Compressor

Figure 2. The structure of RIES integrated with energy storage the specific structure of the base case is shown in Fig. 2.In the case, the ES is connected to point P1. And the power factor of the distribution system is 0.85 . Other key parameters of the two EHs in RIES are shown in Table 1. 
Table 1. Key parameters of the two EHs

\begin{tabular}{|c|c|c|}
\hline Equipment & $\begin{array}{c}\text { Capacity of } \\
\text { equipment }\end{array}$ & $\begin{array}{c}\text { Capacity of } \\
\text { connected pipelines }\end{array}$ \\
\hline $\mathrm{GB}$ & $6 \mathrm{MW}$ & $5 \mathrm{MW}$ \\
\hline $\mathrm{CHP}$ & $\begin{array}{c}\text { Heat:3MW } \\
\text { Electricity: } \\
3 \mathrm{MW}\end{array}$ & $\begin{array}{c}\text { Heat:5MW } \\
\text { Electricity: } \\
5.83 \mathrm{MVA}\end{array}$ \\
\hline $\mathrm{T}_{1}$ & $3 \mathrm{MVA}$ & $5.83 \mathrm{MVA}$ \\
\hline $\mathrm{T}_{2}$ & $5 \mathrm{MVA}$ & $6.91 \mathrm{MVA}$ \\
\hline $\mathrm{ES}$ & $0.5 \mathrm{MW}$ & $5.83 \mathrm{MVA}$ \\
\hline $\mathrm{C}_{1}$ & $5 \mathrm{MW}$ & $0.334 \mathrm{MMCFD}$ \\
\hline $\mathrm{C}_{2}$ & $4 \mathrm{MW}$ & $0.334 \mathrm{MMCFD}$ \\
\hline $\mathrm{T}_{3}$ & $6 \mathrm{MVA}$ & $6.91 \mathrm{MVA}$ \\
\hline $\mathrm{T}_{4}$ & $6 \mathrm{MVA}$ & $6.91 \mathrm{MVA}$ \\
\hline
\end{tabular}

4.2 Two-dimensional visualization and threedimensional visualization of RIESSR integrated with energy storage

Based on interior point method, the TSC solution result of the base case is $16.85 \mathrm{MW}$, and the load distribution of each key pipeline is shown in Table 2 .

Table 2. Load distribution of base case (MW)

\begin{tabular}{|c|c|c|c|c|}
\hline$L_{1}$ & $L_{2}$ & $L_{3}$ & $L_{4}$ & $L_{5}$ \\
\hline 1.2246 & 1.7754 & 2.5500 & 0 & 0 \\
\hline$L_{6}$ & $L_{7}$ & $L_{8}$ & $L_{9}$ & $L_{10}$ \\
\hline 1.0354 & 3.7146 & 2.5500 & 3.9934 & 0.0066 \\
\hline
\end{tabular}

The TSC solution result of the scenario without energy storage is $16.35 \mathrm{MW}$, and the load distribution of each key pipeline is shown in Table 3.

Table 3. Load distribution of the scenario without ES (MW)

\begin{tabular}{|c|c|c|c|c|}
\hline$L_{1}$ & $L_{2}$ & $L_{3}$ & $L_{4}$ & $L_{5}$ \\
\hline 1.2113 & 1.7887 & 2.5500 & 0 & 0 \\
\hline$L_{6}$ & $L_{7}$ & $L_{8}$ & $L_{9}$ & $L_{10}$ \\
\hline 1.0415 & 3.2085 & 2.5500 & 3.9933 & 0.0067 \\
\hline
\end{tabular}

Load combination of key pipeline output $\left(\mathrm{L}_{6}, \mathrm{~L}_{7}\right)$ is selected as the free variable, and other pipeline loads are fixed at TSC operating point. As a comparison, the scenario without ES is established, and other conditions remain unchanged. The two-dimensional security boundaries of two scenarios are solved by fitting critical operating points, which are shown in Fig. 3. And the area of the security region has expanded by approximately $52.5 \%$, of which $27.6 \%$ is cross-quadrant expansion.

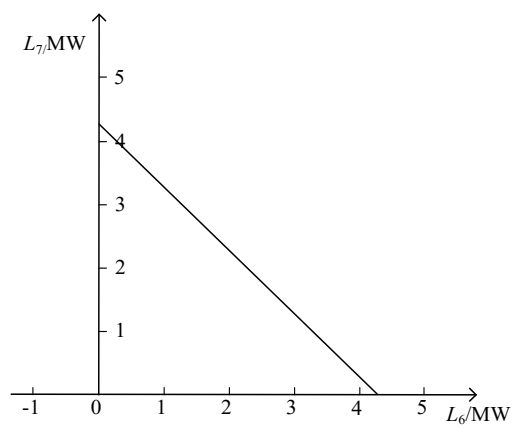

(a) Scenario without ES

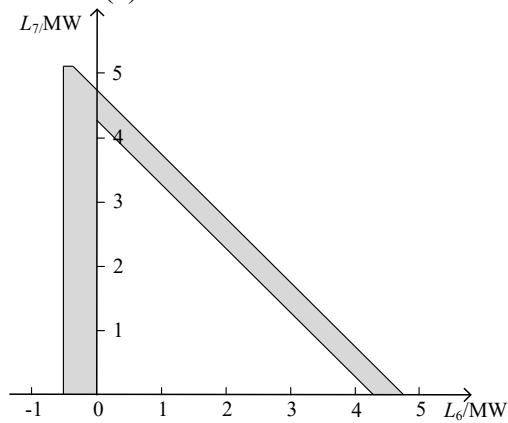

(b) Scenario with ES (0.5MW)

Figure 3 The 2D security boundaries of two scenarios

Then, load combination $\left(\mathrm{L}_{1}, \mathrm{~L}_{2}, \mathrm{~L}_{6}\right)$ is selected as the free variable. The $3 \mathrm{D}$ security boundaries of scenario without ES and scenario with ES are shown in Fig. 4. And the volume of the security region has expanded by approximately $47.3 \%$ across the quadrant.

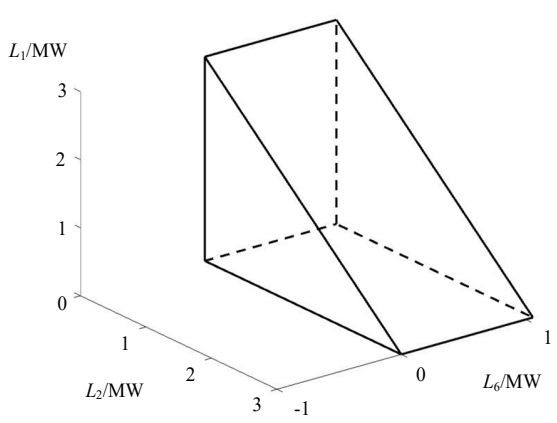

(a) Scenario without ES

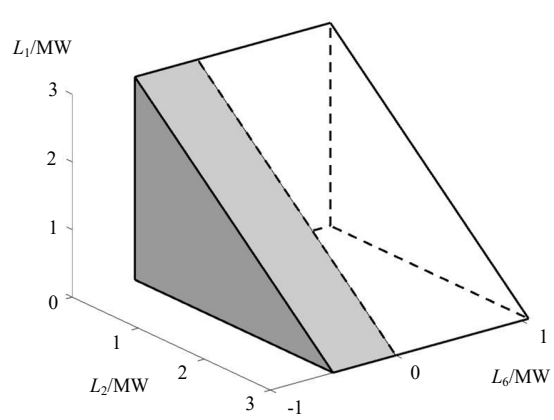

(b) Scenario with ES (0.5MW)

Figure 4 The 3D security boundaries of two scenarios

\subsection{The impact of the energy storage capacity}

Considering the actual project, the capacity of electrical ES can be changed by increasing or decreasing the number of battery packs. Therefore, the capacity of ES is 
a discrete variable. The capacity of a battery pack is set to $0.25 \mathrm{MW}$ in this paper. The TSC solution results is shown in Table 4.

Table 4. TSC of system with different packs of ES

\begin{tabular}{|c|c|c|}
\hline $\begin{array}{c}\text { Number of ES } \\
\text { packs }\end{array}$ & $\begin{array}{c}\text { Capacity of ES } \\
\text { (MW) }\end{array}$ & TSC (MW) \\
\hline 0 & 0 & 16.35 \\
\hline 1 & 0.25 & 16.60 \\
\hline 2 & 0.5 & 16.85 \\
\hline 3 & 0.75 & 17.10 \\
\hline 4 & 1 & 17.20 \\
\hline 5 & 1.25 & 17.20 \\
\hline
\end{tabular}

It can be seen that the TSC increases as the number of ES packs increases. But the TSC on longer increases when the number is more than 4 . Load combinations of key pipeline output $\left(\mathrm{L}_{6}, \mathrm{~L}_{7}\right)$ and $\left(\mathrm{L}_{1}, \mathrm{~L}_{2}, \mathrm{~L}_{6}\right)$ are selected as the free variables. The two-dimensional and threedimensional security boundaries of scenario with 4 packs of ES are shown in Fig. 5. Compared with the scenario without ES, the area of the security region has expanded by approximately $100.5 \%$ in Fig. 5 (a), of which $56.5 \%$ is cross-quadrant expansion. And the volume of the security region has expanded by approximately $100.1 \%$ across the quadrant in Fig. 5 (b).

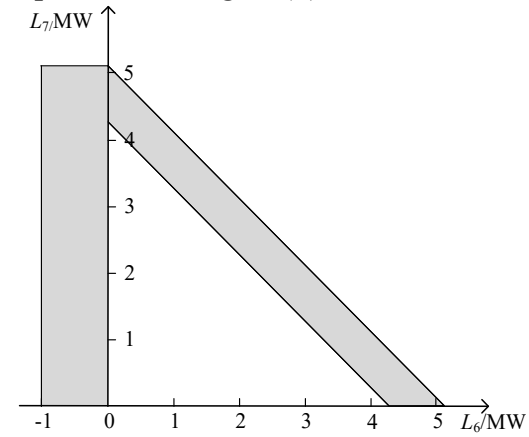

(a) The 2D security boundary

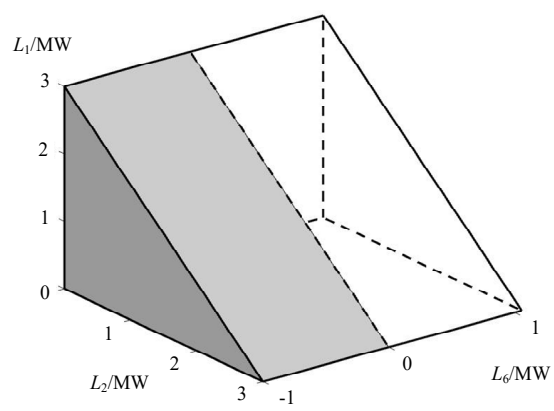

(b) The 3D security boundary

Figure 5 The scenario with ES (1MW)

\subsection{The impact of the energy storage location}

The following part studies the impact of changing the ES location on TSC and RIESSR. In the new scenario, the location of ES is at point P2, which is shown in Fig. 1. And the capacity of the ES is set to $0.5 \mathrm{MW}$. The TSC solution result is $16.35 \mathrm{MW}$. Load combinations of key pipeline output $\left(\mathrm{L}_{7}, \mathrm{~L}_{6}\right)$ and $\left(\mathrm{L}_{1}, \mathrm{~L}_{2}, \mathrm{~L}_{7}\right)$ are selected as the free variables. The security boundaries are shown in Fig. 6. Compared with the scenario without ES, the area of the security region has expanded by approximately $23.5 \%$ in Fig. 6 (a). And the volume of the security region has expanded by approximately $15.6 \%$ in Fig. 6 (b).

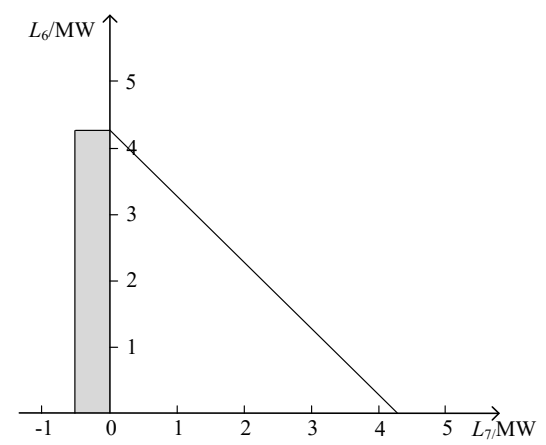

(a) The 2D security boundary

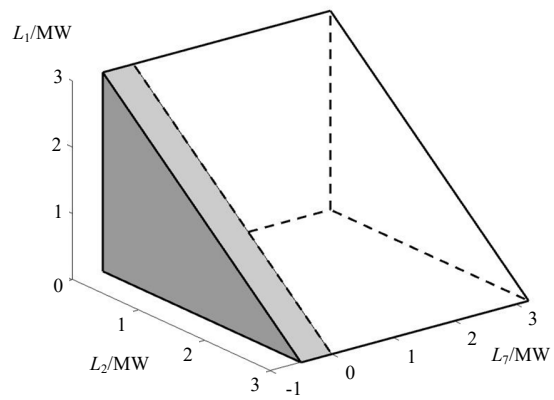

(b) The $3 \mathrm{D}$ security boundary

Figure 6 The scenario with ES (P2)

\section{Conclusion}

By comparing the scenarios, it can be seen that the solution result of TSC increases and the security region extends across quadrant when the RIES is integrated with ES. The reason of expansion across quadrants and improvement of TSC is as follows. Considering the controllability of the ES, its charging process can realize the power backflow to the energy hub and its discharging process can realize the improvement of related equipment energy supply ability.

By increasing the capacity of ES, it can be seen that TSC increases and security region extends with the increase of ES capacity. However, the increase and expansion will stop when the capacity reaches a certain value. This is because the capacity constraints of other key equipment or pipelines have reached the upper limit. By changing the access location, it can be seen that TSC does not increase and the security region only extends across quadrant but not within quadrant when the access location of ES is not appropriate. This is because the capacity of related key equipment and pipelines connected with ES are not the tightest transfer constraints. 


\section{Acknowledgements}

Supported by Open Fund of State Key Laboratory of Power Grid Safety and Energy Conservation (No. YDB51202001922).

\section{References}

1. H. Jia, D. Wang, X. Xu, et al. AUTOMAT ELECTRON POWER SYS. 07, 198 (2015)

2. Y. Yu. J TIANJIN UNIV. 06, 635 (2008).

3. J. Xiao, W. Gu, X. Guo, et al. AUTOMAT ELECTRON POWER SYS. 24, 47 (2011)

4. J. Xiao, Y. Cao, B. Zhang, et al. Transactions of China Electrotechnical Society. 19, 4171 (2020)
5. L. Liu, D. Wang, K. Hou, et al. Appl. Energy. 260 (2020)

6. H. Diao, P. Li, X. Lv, et al. Transactions of China Electrotechnical Society. 01, 151 (2021)

7. Y. Yang, P. Li, Y. Huang, et al. Electric Power. 12, 127 (2020)

8. W. Wang, D. Wang, H. Jia, et al. Proceedings of the CSEE. 12, 3292 (2016)

9. L. Liu, D. Wang, H. Jia, et al. Electric Power Automation Equipment, 08, 63 (2019)

10. S. Chen, Z. Wei, G. Sun, et al. Electric Power Automation Equipment, 08, 3 (2019)

11. M. Abeysekera, J. Wu, N. Jenkins, et al. Appl. Energy. 164, 991 (2015) 